\title{
Macrophages yo-yo during weight loss
}

Obesity is associated with the accumulation of macrophages in adipose tissues (fat), where these cells contribute to local and systemic inflammation and the modulation of metabolic processes. But until now, no careful analysis of the macrophage response to weight loss had been carried out. Ferrante and colleagues show that macrophage recruitment to adipose tissues initially increases following weight loss and then declines, in line with the levels of free fatty acids (FFAs) in the blood. The findings suggest that lipolysis (the metabolism of triglycerides into FFAs) drives the accumulation of macrophages in adipose tissue; the macrophages then take up lipids to control local lipid concentrations.

Analysis of macrophage dynamics and gene expression over a period of gradual weight loss in obese mice on a calorie-restricted diet indicated that initially (after 3-7 days of calorie restriction) macrophage numbers in adipose tissues increased and then (by 60 days) decreased to levels lower than those present prior to the start of weight loss. Unexpectedly, the initial increase in macrophage accumulation was not associated with a concomitant increase in inflammatory gene expression, suggesting that non-inflammatory macrophages are recruited to the adipose tissues soon after the initiation of weight loss.
The initial weight loss-induced increase in adipose tissue macrophages was associated with higher concentrations of FFAs in the serum. FFAs are released from adipocytes during basal lipolysis (which occurs at a higher rate in obese individuals) and during demand lipolysis (which is induced during periods of increased energy demand). During initial dieting, basal lipolysis remains high and demand lipolysis increases, leading to a net increase in FFA levels, which is consistent with the observed increase in expression of the lipolysis enzyme adipose triglyceride lipase (ATGL; also known as PNPLA2). The authors observed a positive correlation between adipose tissue lipolysis and macrophage numbers. This correlation was observed in obese mice on a calorie-restricted diet, as well as in lean mice that were fasted for 24 hours. Accordingly, pharmacological induction of lipolysis led to a rapid accumulation of adipose tissue macrophages, and mice that lack ATGL expression (and therefore have impaired lipolysis) showed no increase in adipose tissue macrophages after fasting.

Macrophages that were recruited to adipose tissue during the initial period of weight loss had increased expression of the lipid transport recep-

tors CD36 and macrophage scavenger receptor 1 , suggesting that they may function in lipid uptake. Indeed, the authors observed higher levels of lipid droplet formation and lipid-containing vesicles in adipose tissue macrophages from mice that underwent 24 hour fasting than from control mice. This function of adipose tissue macrophages was shown to contribute to the regulation of local lipid concentrations: mice that were depleted of macrophages and then fasted for 24 hours had further increases in serum FFA levels compared with control mice.

So, similar to the role of macrophages in controlling local excess cholesterol in the arteries, macrophages can respond to acute changes in lipolysis and migrate to adipose tissues, where they phagocytose excess lipids without causing inflammation. As triglyceride stores are depleted and basal lipolysis decreases during ongoing weight loss, macrophages leave the adipose tissue and metabolic function is protected.

Lucy Bird

ORIGINAL RESEARCH PAPER Kosteli, A. et al. Weight loss and lipolysis promote a dynamic immune response in murine adipose tissue. J. Clin. Invest. 120, 3466-3479 (2010) 